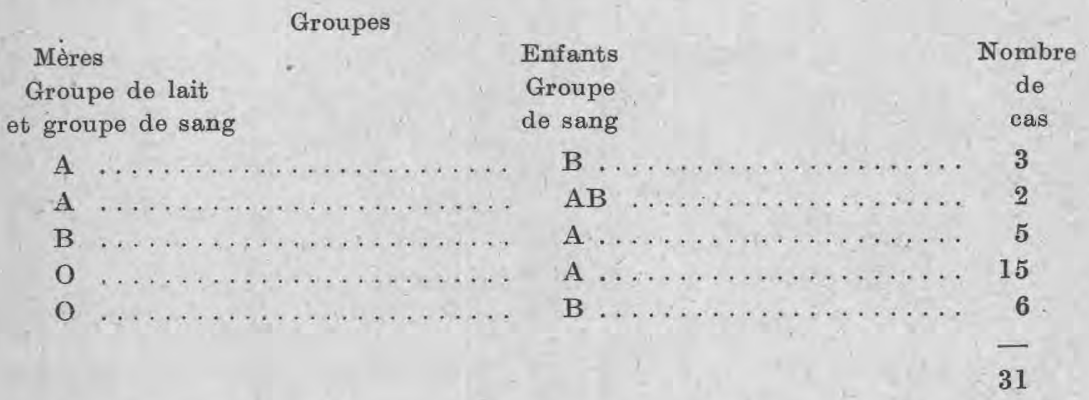

Ces cas sont trop peu nombreux pour permettre de tirer une conclusion, mais ils le sont assez pour justifier de nouvelles recherches.

Le problème ne pourra être résolu que par des recherches simultanées de clinique et de sérologie.

\title{
REVUE
}

\section{LES PROGRÈS, EN ITALIE, DE LA FABRICATION DES FIBRES SYNTHÉTIQUES EN PARTANT DE CASÉINE}

\author{
par \\ G. GÉNIN \\ Ingénieur E. P. C.
}

Nous avons parlé à plusieurs reprises déjà de la fabrication, en Italie, d'une fibre synthétique connue sous le nom de "lanital ", et préparée en partant de caséine. C'est en 1935 que les premiers brevets furent pris par A. FerRetri; en septembre de la même année, un atelier expérimental était construit dans les usines de la "Snia Viscosa", à Ceseano Maderno (près de Milan), et dès novembre 1935, on trouvait sur le marché italien les premiers échantillons de lanital. On sait aujourd'hui qu'une nouvelle usine va être prochainement mise en route à Ceseano Maderno, ce qui portera la capacité totale de production de lanital de ces deux usines à environ 30.000 kilogrammes par jour. On sait également que d'autres pays, en particulier la France, le Canada, la Belgique, la Hollande, la Pologne et l'Allemagne ont acquis les licences du procédé Ferretti.

De nouvelles informations nous sont parvenues sur la fabrication de ce produit qui compléteront celles que nous avons données à plusieurs reprises.

La caséine provenant des différents centres producteurs est 
recueillie dans des réservoirs séparés et particuliers à chaque centre, et on mélange les lots différents en proportions bien déterminées, pour obtenir un mélange aussi uniforme que possible. Le mélange est alors placé dans des réservoirs et on lui ajoute de l'eau et certains produits qui permettront d'obtenir une pâte visqueuse qui servira au filage de la fibre artificielle.

\section{Filage et traitement par la formaldéhyde}

Le filage s'effectue en obligeant la pâte à traverser des filières dans lesquelles on a percé un nombre important de petits trous ayant de $0 \mathrm{~mm} .02$ à $0 \mathrm{~mm}$. 03 de diamètre. Les fils très fins ainsi obtenus passent dans une solution chaude d'acide sulfurique dans laquelle la caséine se coagule et durcit. L'acide contenu dans les fibres est alors neutralisé en les faisant passer dans un bain alealin, après quoi on découpe les fibres en très petits morceaux qui forment des "flocks ». Finalement, ces petites brindilles sont immergées dans un bain de formaldéhyde dans lequel elles restent de 10 à 15 heures. On procède ensuite au séchage dans des machines chauffées à la vapeur et le produit est prêt à être filé, puis tissé.

Nous rappellerons les résultats d'analyse chimique effectuée sur des échantillons de lanital qui sont les suivants : carbone, $53 \%$; hydrogène, $7 \%$; oxygène, $23 \%$; azote, $15,5 \%$; soufre, $0,7 \%$; divers, $0,8 \%$. En comparant ces chiffres aux résultats d'analyse de la laine naturelle : carbone, $49,25 \%$; hydrogène, $7,57 \%$; oxygène, $23,66 \%$, azote, $15,86 \%$, soufre, $3,66 \%$, on constatera que la proportion de soufre est nettement moins élevée dans le produit artificiel que dans le produit naturel et e'est pourquoi les propriétés isolantes du lanital sont supérieures à celles de la laine naturelle, cette propriété étant en sens inverse de la teneur en soufre.

\section{Lavage et teinture.}

Les derniers échantillons de lanital fabriqués en Italie, et qui ont par conséquent pu bénéficier des améliorations apportées à cette fabrication ont été examinés au point de vue de leur résistance au lavage. Un échantillon de tissu de lanital que l'on fait bouillir dans de l'eau pure pendant 3 heures ne perd pas de poids. Le même produit porté à l'ébullition pendant le même temps dans une solution fortement alcaline de savon ne perd qu'un poids inférieur à celui constaté sur un échantillon de tissu de laine mérinos soumis au même traitement. Les fibres et les tissus de lanital sont facilement teints par les colorants acides.

L'usine initiale qui est encore aujourd'hui en fonctionnement, a une production journalière de 5.000 kilogrammes de lanital qui est entièrement absorbée par l'industrie textile du pays. La nouvelle usine, voisine d'ailleurs de la précédente, pourra produire 25.000 kilo- 
grammes de lanital par jour. Tout le matériel nécessaire à cette fabrication a d'ailleurs été construit dans les ateliers de la "Snia Viscosa », de Turin, qui construisent également le matériel destiné à la fabrication de la soie artificielle.

\section{La question des matières premières.}

On a indiqué que les prix de vente des fibres Lanital seraient de 20 à 21 lires par kilogramme, ce qui laisserait un bénéfice raisonnable au fabricant. Or, le prix de la laine naturelle sur le marché international est légèrement plus élevé et s'établit à environ 25 lires le kilogramme, mais sur le marché italien, les cours sont beaucoup plus élevés.

Quant au prix de la caséine en Italie qui était de 2 à 3 lires le kilogramme, avant que la fabrication du lanital fût commencée, il a maintenant doublé sous l'influence de la loi de l'offre et de la demande qui semble done jouer tout aussi bien sous un gouvernement dictatorial que sous un gouvernement démocratique.

On pense que la production totale de caséine en Italie est d'environ 10 millions de kilogrammes par an, mais la totalité de cette caséine n'est pas encore utilisée pour la fabrication du lanital. Actuellement, les centres collecteurs de la "Snia Viscosa " recueillent annuellement 1.500 .000 kilogrammes à 2 millions de kilogrammes de caséine. Peu à peu, cette organisation se développera et on peut penser qu'un jour elle tendra, peu à peu, à atteindre le maximum annuel de 10 millions de kilogrammes. A ce moment, l'Italie fabriquerait une quantité suffisante de lanital pour assurer 20 à $25 \%$ de ses besoins en laine, sans compter la laine qu'elle utilise pour alimenter son commerce d'exportation.

En ce qui concerne la caséine importée, son cours est, en Italie, légèrement inférieur à 6 lires le kilogramme et la “Snia Viscosa» fait déjà d'importants achats de caséine en Hollande et au Danemark, et étudie la possibilité d'en acheter en Argentine.

L'industrie textile italienne fabrique déjà un grand nombre de tissus de tous types, de toutes qualités et de toutes couleurs, en mélangeant, en proportions variables, du lanital et de la laine naturelle. Des études sont également en cours, afin d'entreprendre la fabrication de fibres complexes obtenues par mélange de lanital et de fibres cellulosiques ; par exemple, on peut ajouter à la solution colloïdale de caséine un pourcentage déterminé d'une solution colloïdale de viscose, normalement employée dans la fabrication de la rayonne; l'addition est faite de préférence quelques heures avant la filature. En faisant varier la proportion de cellulose, on obtient toute une gamme de fibres textiles artificielles et A. 
Ferretri a proposé les noms suivants pour désigner ces différentes fibres :

a) "Serinlaine " quand le pourcentage de caséine est supérieur au pourcentage de cellulose; les fibres textiles ainsi obtenues contiennent au moins $7 \%$ d'azote.

b) "Rayonlaine» lorsque le pourcentage de caséine est inférieur à celui de la cellulose, la teneur en azote étant alors inférieure à $7 \%$.

Pour terminer, nous indiquerons que le brevet français de A. FerRetti vient d'être publié(1) il y a quelques semaines seulement, il porte le no 813.427 ; il a été déposé le 26 août 1936 à l'Office de la propriété industrielle, avee priorité italienne du 28 août 1935 .

\section{BIBLIOGRAPHIE ANALYTIQUE}

\section{LES LIVRES}

Eight Annual Report. The Hannah Dairy Research Institute. Annual Report ( $8^{e}$ Rapport annuel de l'Institut de Recherches laitières de Hannah). (Année se terminant le 31 mars 1937.)

\section{RECHERCHES BIOLOGIQUES}

Besoins en protéine des vaches laitières.

Depuis six ans, l'Institut étudie cette question. Il s'agit de déterminer si les standards alimentaires actuels ne pourraient pas être diminués sans influencer la santé des animaux ou diminuer leur rendement laitier, et si on ne pourrait pas remplacer des quantités importantes des produits concentrés importés par des produits alimentaires indigènes.

Les résultats obtenus prouvent que les besoins en protéine pour la production du lait peuvent être réduits d'au moins un quart (c'est-à-dire de $0 \mathrm{~kg}$. 6 à $0 \mathrm{~kg}$. 45 de protéine, équivalant à $10 \mathrm{~kg}$. de lait) si les rations sont judicieusement choisies. Le dernier chiffre indiqué a été utilisé pendant plusieurs années pour l'alimentation du troupeau de l'Institut sans effets désagréables, ni sur la santé des animaux, ni sur le rendement laitier du bétail. En outre, il a été prouvé que la valeur biologique des protéines des divers aliments diffèrent et qu'en général les protéines provenant des aliments indigènes (poudre de sang fraîche ou séchée, herbe ensilée, farine de fèves) ont une valeur alimentaire plus élevée que celles provenant des concentrés importés, telle que les tourteaux d'arachide et de lin.

Production de fourrage séché artificiellement.

Deux appareils de séchage convenant à une ferme laitière d'importance moyenne (appareils Ransome et P. et M.) fonctionnent à l'Institut depuis deux ans. Un certain nombre de difficultés ont été rencontrées dans leur utilisation, et il n'est pas encore possible de dire si on peut produire, dans des

(1) Voir p. 519. 